
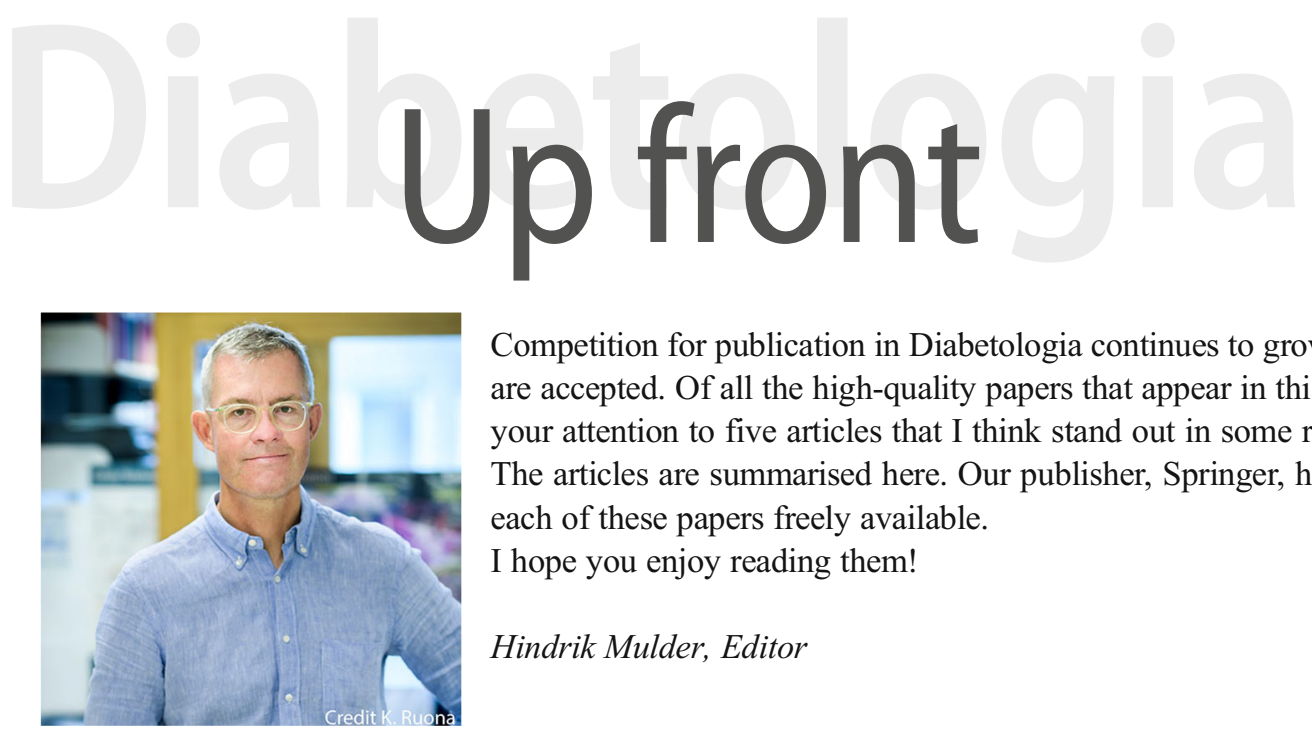

Competition for publication in Diabetologia continues to grow, and less than $20 \%$ of papers are accepted. Of all the high-quality papers that appear in this month's issue I want to draw your attention to five articles that I think stand out in some regard and are very interesting. The articles are summarised here. Our publisher, Springer, has kindly made the full text of each of these papers freely available.

I hope you enjoy reading them!

Hindrik Mulder, Editor

\section{Metabolic, structural and biochemical changes in diabetes and the development of heart failure}

Kim L. Ho, Qutuba G. Karwi, David Connolly, Simran Pherwani, Ezra B. Ketema, John R. Ussher, Gary D. Lopaschuk.

Diabetes increases the risk of heart failure by over two-fold, and the need to improve our understanding of the way in which this occurs is becoming ever important. In this issue, Ho et al (https://doi.org/10.1007/s00125-021-05637-7) summarise the cardiac metabolic, structural, and biochemical changes that occur in diabetes. The authors discuss how hyperlipidaemia and hyperglycaemia contribute to changes in fatty acid and glucose metabolism, and that structural remodelling of the heart occurs in the form of hypertrophy and fibrosis. They go on to explore how, biochemically, impairments in calcium handling, glucotoxicity, lipotoxicity, and transcriptional and translational modifications all contribute to cardiac dysfunction in diabetes. The authors conclude by highlighting that with the increasing amount of research going into the mechanisms by which certain diabetes drugs improve cardiovascular outcomes, new therapeutic strategies are emerging to treat diabetes and heart failure. The figures from this review are available as a downloadable slideset.

Artificial intelligence utilising corneal confocal microscopy for the diagnosis of peripheral neuropathy in diabetes mellitus and prediabetes

Frank G. Preston, Yanda Meng, Jamie Burgess, Maryam Ferdousi, Shazli Azmi, Ioannis N. Petropoulos, Stephen Kaye, Rayaz A. Malik, Yalin Zheng, Uazman Alam.
The accurate detection of diabetic neuropathy in routine clinical practice remains a major unmet clinical need. Current screening practices largely rely on insensitive tests which only primarily detect the insensate foot. It has been demonstrated that artificial intelligence (AI) trained using annotated corneal confocal microscopy images can provide accurate segmentation of corneal nerve images, allowing the detection of peripheral neuropathy. In this issue, Preston and Meng et al (https://doi.org/10.1007/ s00125-021-05617-x) report that AI utilising corneal nerve images can accurately classify peripheral neuropathy in people with prediabetes and diabetes, without the need for underlying nerve segmentation. This was achieved by use of a single corneal confocal microscope image. The authors discuss that, as annotation of the image/dataset was not required, larger sets of unannotated images may be leveraged in providing a more robust model. The authors conclude that with validation in a larger realworld study, the AI algorithm has considerable potential for adoption into screening programmes for diabetic neuropathy.

\section{Multi-ethnic GWAS and fine-mapping of glycaemic traits} identify novel loci in the PAGE Study

Carolina G. Downie, Sofia F. Dimos, Stephanie A. Bien, Yao Hu, Burcu F. Darst, Linda M. Polfus, Yujie Wang, Genevieve L. Wojcik, Ran Tao, Laura M. Raffield, Nicole D. Armstrong, Hannah G. Polikowsky, Jennifer E. Below, Adolfo Correa, Marguerite R. Irvin, Laura J. F. Rasmussen-Torvik, Christopher S. Carlson, Lawrence S. Phillips, Simin Liu, James S. Pankow, Stephen S. Rich, Jerome I. Rotter, Steven Buyske, Tara C. Matise, Kari E. North, Christy L. Avery, Christopher A. Haiman, Ruth J. F. Loos, Charles Kooperberg, Mariaelisa Graff, Heather M. Highland. 
Previous genome-wide association studies (GWAS) have identified over 500 loci associated with type 2 diabetes and glycaemic-related traits. However, most of these studies were conducted in populations of European ancestry. This lack of ancestral diversity hinders efforts to identify novel loci, refine causal signals through fine-mapping, and develop equitable genetic approaches for precision medicine and risk prediction. In this issue, Downie et al (https://doi.org/10.1007/s00125021-05635-9) identify novel glycaemic trait loci in the ancestrally diverse Population Architecture using Genomics and Epidemiology (PAGE) Study. Specifically, the authors identify three fasting insulin novel loci in a transethnic metaanalysis, one novel low-frequency fasting glucose locus in an African American-specific analysis, and novel independent secondary signals at known fasting glucose and insulin loci. The authors conclude that these findings highlight the continued importance of conducting genetic studies in diverse populations and provide new insights into the genetic architecture of glycaemic traits.

Dietary carbohydrate restriction augments weight lossinduced improvements in glycaemic control and liver fat in individuals with type 2 diabetes: a randomised controlled trial

Mads N. Thomsen, Mads J. Skytte, Amirsalar Samkani, Martin H. Carl, Philip Weber, Arne Astrup, Elizaveta Chabanova, Mogens Fenger, Jan Frystyk, Bolette Hartmann, Jens J. Holst, Thomas M. Larsen, Sten Madsbad, Faidon Magkos, Henrik S. Thomsen, Steen B. Haugaard, Thure Krarup.

Weight loss is the cornerstone of management of type 2 diabetes. Whether a diet reduced in carbohydrate and increased in protein and fat can augment the beneficial effects of weight loss, compared with a conventional diabetes diet, is not known. In this issue, Thomsen et al (https://doi.org/10.1007/ s00125-021-05628-8) present the results of a randomised study that evaluated the metabolic effects of matched $6 \%$ weight loss, induced after 6 weeks of a fully-provided carbohydrate-reduced diet (30\% of energy from carbohydrate) or a conventional diabetes diet (50\% of energy from carbohydrate) in individuals with type 2 diabetes. The authors show that the experimental diet was well tolerated and, for the same amount of weight loss as that induced by the control diet, augmented the reduction in $\mathrm{HbA}_{1 \mathrm{c}}$ by $1.9 \mathrm{mmol} / \mathrm{mol}$, in liver fat by $26 \%$, in plasma triacylglycerol by $18 \%$ and in diurnal blood glucose by $0.8 \mathrm{mmol} / \mathrm{l}$. The authors conclude that carbohydrate reduction has weight loss-independent beneficial metabolic effects and should be considered in the treatment of type 2 diabetes.

Wt1 haploinsufficiency induces browning of epididymal fat and alleviates metabolic dysfunction in mice on highfat diet

Karin M. Kirschner, Anna Foryst-Ludwig, Sabrina Gohlke, Chen Li, Roberto E. Flores, Ulrich Kintscher, Michael Schupp, Tim J. Schul, Holger Scholz.

The induction of thermogenically active beige adipocytes in white adipose tissue (WAT) is a key feature of WAT browning. Browning has recently gained interest for its potential use to enhance energy expenditure. Beige adipocytes can be readily induced in subcutaneous WAT of mice, whereas visceral WAT is more resistant to browning. In this issue, Kirschner et al (https://doi.org/10.1007/ s00125-021-05621-1) show that otherwise healthy mice, heterozygous for the Wilms tumour gene, Wt1, display morphological and genetic signs of browning in their visceral WAT. Strikingly, Wt1 heterozygosity improved whole-body glucose tolerance and prevented severe hepatic steatosis under a high-fat diet. Mechanistically, the authors identified WT1 as an upstream regulator of Aldhla1 and Zfp423, key suppressors of the thermogenic programme in adipocytes. Their data provide evidence that WT1 downregulates thermogenic genes and functions as a white adipocyte determination factor in visceral WAT. The authors conclude that targeting Wt1 expression in visceral fat may offer a promising novel approach to fight metabolic disorders.

Publisher's note Springer Nature remains neutral with regard to jurisdictional claims in published maps and institutional affiliations.

All text supplied by the authors. 